\title{
Oral low-dose estradiol plus norethisterone acetate with or without simvastatin in dyslipidemic and symptomatic menopausal women: A randomized clinical trial ${ }^{*}$
}

\author{
Marcelo Luis Steiner ${ }^{1 \#}$, Luciano Melo Pompei ${ }^{2}$, Rodolfo Strufaldi ${ }^{3}$, Everaldo Porto Cunha ${ }^{3}$, \\ César Eduardo Fernandes ${ }^{4}$ \\ ${ }^{1}$ Postgraduate Student in Gynecology, Obstetricsand Mastology, Universidade Estadual de São Paulo (Unesp), Botucatu, São Paulo, \\ Brazil; " Corresponding Author: masteiner@ig.com.br \\ ${ }^{2}$ Assistant Physician, Gynecological Endocrinologyand Menopause Division, Department of Gynecology and Obstetrics, Faculdade \\ de Medicina do ABC (FMABC), São Bernardo do Campo, São Paulo, Brazil \\ ${ }^{3}$ Assistant Professor, Department of Gynecologyand Obstetrics, Faculdade de Medicina do ABC (FMABC), São Bernardo do Campo, \\ São Paulo, Brazil \\ ${ }^{4}$ Head of the Gynecological Endocrinology and Menopause Division, Department of Gynecology and Obstetrics, Faculdade de Medi- \\ cina do ABC (FMABC), São Bernardo do Campo, São Paulo, Brazil
}

Received 9 May 2013; revised 11 June 2013; accepted 15 July 2013

Copyright (C) 2013 Marcelo Luis Steiner et al. This is an open access article distributed under the Creative Commons Attribution License, which permits unrestricted use, distribution, and reproduction in any medium, provided the original work is properly cited.

\section{ABSTRACT}

Context and Objective: Cardiovascular diseases are the leading causes of death in postmenopausal women and dyslipidemia has important contributing factor. The objective of the study was to evaluate low-dose estrogen plus progestogen therapy (EPT) + simvastatin for vasomotor symptoms and lipid and lipoprotein profiles. Design and Setting: Clinical trial was conducted in a private clinical research center. Methods: Two hundred forty symptommatic postmenopausal women with dyslipidemia were randomized to one of three treatment groups: A) $1 \mathrm{mg}$ estradiol/ $0.5 \mathrm{mg}$ norethisterone acetate (E2/NETA) + 20 mg simvastatin; B) E2/NETA + placebo; or C) 20 mg simvastatin + placebo. Lipid and lipoprotein profiles and menopausal symptoms were evaluated after 16 weeks. Results: Total cholesterol, LDL cholesterol, non-HDL cholesterol decreased $(P<0.05)$ in all three groups, Apo-B/Apo-A1 and ApoB $(P<0.01)$ in groups $A$ and $C$ and $T G$ and ApoA1 only in group $A$ comparing baseline versus final. Comparing the groups, group $B$ showed differences in relation to others in total

*Instituto de Saúde e Bem Estar da Mulher (ISBEM), São Paulo, São Paulo, Brazil.

Sources of funding: None

Conflict of interest: None cholesterol, non-HDL cholesterol, LDL cholesterol, Apo B and ApoB/Apoa1 $(P<0.01)$. Relief of menopausal symptoms was better compared in $A$ and B. Conclusions: Thus in postmenopausal women with dyslipidemia, the association of E2I NETA low-dose with simvastatin relieved climacteric symptoms similar to that observed with isolated E2/NETA and improved lipid and lipoprotein profile similar to the isolated use of simvastatin. The use of E2/NETA alone decreased menopausal symptoms, but did not improve dyslipidemia.

Keywords: Dyslipidemia; Hormone Replacement Therapy; Lipoproteins; Simvastatin; Cardiovascular Diseases

\section{INTRODUCTION}

Menopause heralds a time of new challenges for women, particularly with respect to the menopausal symptoms that affect quality of life and the increased cardiovascular risk. The most prevalent of these symptoms are the hot flushes that affect more than $60 \%$ of menopausal women. At this same time, cardiovascular diseases (CVD) become the principal culprits responsible for mortality in women of this age-group [1,2].

Postmenopausal hormone therapy (HT) is the most ef- 
fective treatment for menopausal symptoms. Based on observational studies, HT was indicated for the primary and secondary prevention of CVD and was long recommended as the treatment of choice for menopausal women [2-4]. Nevertheless, following interventional studies who questioned the benefits of replacement therapy for cardiovascular disease, $[5,6]$ physicians became more reluctant to prescribe this therapy, despite the methodological issues involved in these studies [7].

On the other hand, statins have been shown to represent one of the good options in the therapeutic arsenal for cardiovascular disease [8-10]. Their capacity to combat dyslipidemia, together with their antiinflammatory and antioxidant effects that stabilize atheromatous plaques decreasing the likelihood of rupture, appear to be the principal mechanisms responsible for this beneficial effect $[11,12]$. Nevertheless, it is not yet known whether this drug exerts any effect on menopausal symptoms. Various studies have shown the positive effect of HT on the lipid profile [13-16]. However, in view of the negative evidence on cardiovascular risk, this benefit does not support the recommendation of HT for the primary prevention of CVD.

Therefore, one of the options for the use of HT in symptomatic menopausal women with dyslipidemia without fear of increasing cardiovascular risk would be to associate this therapy with a statin. It is believed that the result of this combination would be positive and would lead to a decrease in the risk of cardiovascular events such as acute myocardial infarction and cerebrovascular accidents in postmenopausal women.

Fak et al. evaluated this drug combination and concluded that it was more effective than simvastatin alone for the treatment of hypercholesterolemia [15]. However, they used high-dose HRT and failed to evaluate cardiovascular risk markers. To the best of our knowledge, there are no studies in the literature showing the benefits of low-dose HRT in association with simvastatin on lipid and lipoprotein profile. Therefore, the objective of the present study was to evaluate the effects of treatment with low-dose postmenopausal hormone therapy alone or in combination with simvastatin on lipids and lipoproteins profile in symptomatic postmenopausal women.

\section{METHODS}

A prospective, parallel, randomized (1:1:1), doubleblind, placebo-controlled study was conducted in a private clinical research center. All the procedures carried out in the study were previously approved by the institute's internal review board. All the participants signed an informed consent form prior to inclusion in the study.

A total of 964 women from different primary care centers of São Paulo city recruited in the ISBEM's (Instituto de Saúde e BemEstar da Mulher) data base were invited to participate in the trial. Inclusion criteria consisted of: age 45 - 65 years, amenorrhea for $\geq 12$ months, Kupperman Index $(\mathrm{KI}) \geq 8$ points (total possible score: 51 ), and serum total cholesterol (TC) levels $>200 \mathrm{mg} / \mathrm{dl}$ and $<320 \mathrm{mg} / \mathrm{dl}$ or LDL $>130 \mathrm{mg} / \mathrm{dl}$ and $<190 \mathrm{mg} / \mathrm{dl}$ or triglycerides (TG) between $150 \mathrm{mg} / \mathrm{dl}$ and $400 \mathrm{mg} / \mathrm{dl}$.

The exclusion criteria comprised: a) hysterectomized women; b) known hypersensitivity to simvastatin, estradiol or norethisterone acetate; c) body mass index (BMI) $\geq 35 \mathrm{~kg} / \mathrm{m}^{2}$; d) cytological and colposcopic evidence of cervical atypia within the past 12 months; e) transvaginal ultrasonography performed within the past six months showing endometrial thickness $>10 \mathrm{~mm}$; f) mammography within the previous six months classified as BI$\operatorname{RADS}^{\circledR} 0,4,5$ or 6 ; g) liver disease or ALT and AST levels above the upper limits of normal; h) diabetes mellitus or fasting glucose $\geq 126 \mathrm{mg} / \mathrm{dl}$; i) personal history of CVD or thromboembolism; j) previous or current hormone-dependent tumors; and k) uncontrolled hypothyroidism $(\mathrm{TSH}>5.0 \mu \mathrm{IU} / \mathrm{ml})$.

As shown in Figure 1, 242 of the women invited to participate in the study met the admission criteria and attended visit 1 (V1). Baseline $\mathrm{KI}$ and serum levels of TG, TC, HDL, LDL, TSH, apolipoproteins A1 and B (ApoA1 and Apo-B), creatine kinase (CK), creatinine, glucose and transaminases were assessed. Next, the women were randomized and allocated to the treatment groups.

Treatment was conducted over 16 weeks, with the women visiting the clinic at 8 weeks (V2) and after the 16 weeks of treatment (V3). The laboratory tests and the KI evaluation were repeated at V2 and V3.

\subsection{Treatment}

Medication used in the study consisted of: a tablet of 1 $\mathrm{mg}$ estradiol/0.5 mg norethisterone acetate (E2/NETA) (Biolab Farmacêutica, São Paulo, Brazil) and a tablet of 20 mg simvastatin (Biolab Farmacêutica, São Paulo, Brazil). The placebos (Biolab Farmacêutica, São Paulo, Brazil) used were identical to the active drugs in appearance, consistency, size, taste and smell. Patients were instructed to take all medication at night. All patients were followed by a single physician who was responsible for the attendance at all visits.

The patients included in the study were randomly assigned to one of three treatment groups: Group A: patients received E2/NETA + simvastatin; Group B: patients received E2/NETA + the simvastatin placebo; and Group C: patients received the E2/NETA placebo + simvastatin.

The adherence to study treatment was checked in all visits by counting the returned tablets. Those women 


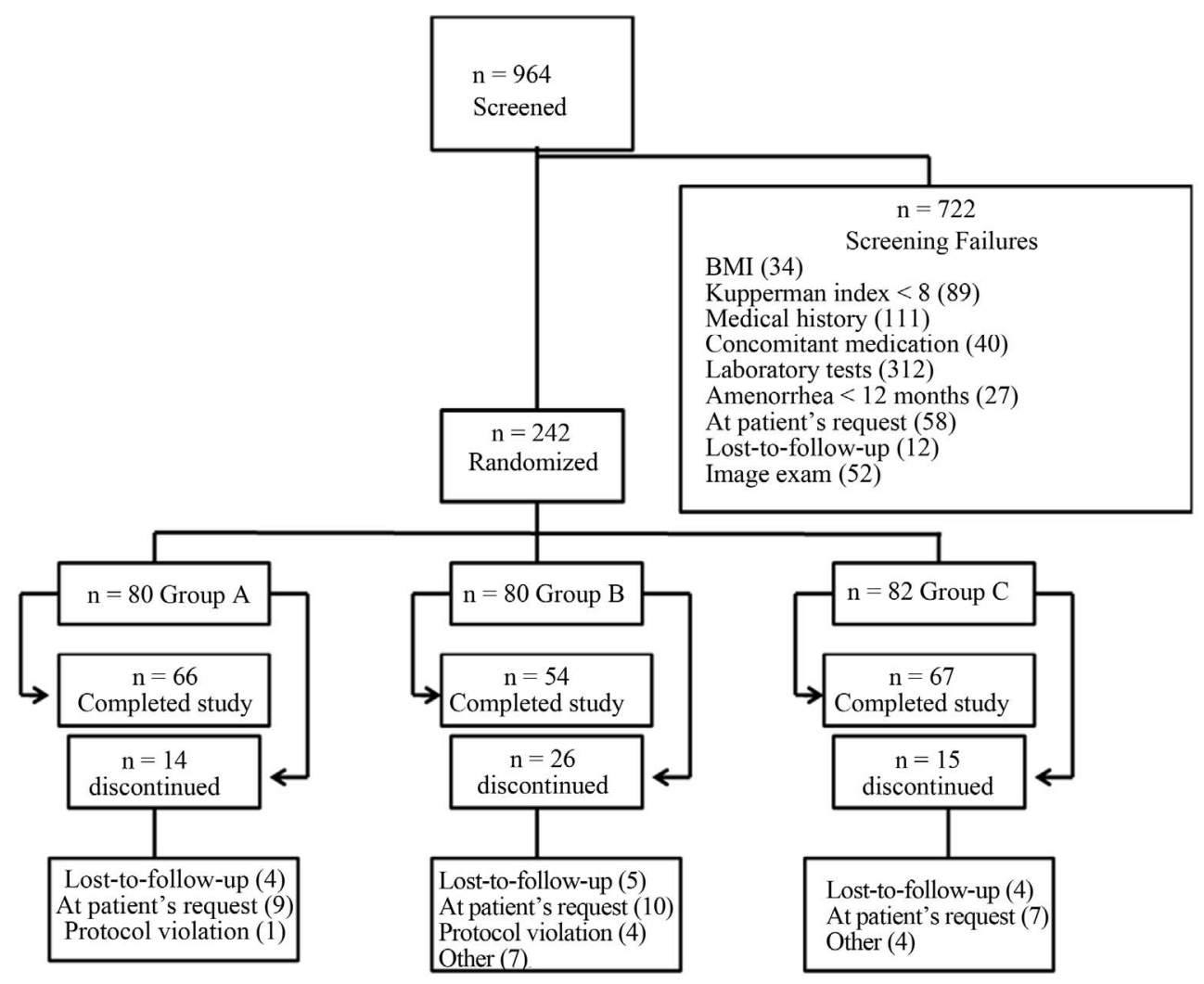

Figure 1. Flowchart of patients in the study.

who failed to make use of three consecutive days or more and those who had a treatment adherence less than $20 \%$ were excluded.

\subsection{Efficacy Parameters}

The treatment was considered effective for menopausal symptoms when there was improvement in symptoms at final visit compared to baseline. The efficacy of the treatment on dyslipidemia was measured according to whether an improvement was found in the lipid profile, as shown by a reduction in TG, TC and LDL or an increase in HDL at the final visit compared to baseline. The efficacy of the treatment on the cardiovascular risk markers was determined by an increase in Apo-A1, a reduction in Apo-B or a reduction in Apo-B/Apo A1 ratio when values at the final visit were compared with baseline.

Blood samples were drawn in the morning after a 12-hour fast at ISBEM by a trained nurse. The handling and storage of samples was done according to clinical laboratory's guidelines. All the biochemical tests were assayed on Byosystems A15 autoanalyzer (Biosystems S.A, Costa Brava 30, Barcelona, Spain). Measurements of the creatine kinase, creatinine, glucose and transaminases were performed by a Biosystemsturbidimetry assay (A15, Biosystems, Barcelona, Spain), while TC, TG and
HDL were spectrophotometrically enzymatic measured using Biosystem kits (Biosystems, Barcelona, Spain). LDL levels were obtained by subtracting TG divided by 5 and HDL from the TC level (Friedwald's formula). Turbidimetry was also used to quantify Apo-A1 and ApoB (Biosystems, Barcelona, Spain).

\subsection{Randomization}

The randomization method was block randomization performed by Random Allocation Software, version 1.0.0, 2004 , in a single session. The women were randomized to the tree different groups $(1: 1: 1)$ according to a list generated by the program. After the patients had been assigned to the three groups, the order of random drug dispensing was tested, also at a 5\% level, using the WaldWolfowitz test.

\subsection{Calculation of Sample Size}

Sample size calculation used as a reference results of Hsia J. et al. study who noted a standard deviation of 40 $\mathrm{mg} / \mathrm{dl}$ total cholesterol [17]. Using the "Power and Sample Size Program" software, version 2.1.31, Dupont and Plummer estimated that from a sample of 66 in each treatment group should identify a difference between groups in total cholesterol $24 \mathrm{mg} / \mathrm{dl}$, with a power $80 \%$ and a type I error (alpha) of 1\% [18]. Considering 20\% 
of drop-outs was considered an ideal of 80 patients per group. This sample also has the ability to detect an intragroup difference of $14 \mathrm{mg} / \mathrm{dl}$ in total cholesterol.

\subsection{Statistical Analysis}

The baseline data of all randomized patients were considered in the final analysis (intention to treat analysis). However, in the evaluation of the efficacy of treatment, only the data from those women who had used the medication correctly at least $80 \%$ of the time were taken into consideration (per protocol analysis).

To evaluate comparability between the treatment groups, within-group comparisons, the demographic characteristics, baseline anthropometric data and laboratory tests of the population were compared using analysis of variance (ANOVA).

The KI was evaluated using analysis of variance for the mean of the variations between the basal visit versus $\mathrm{V} 2$ and basal versus final visit. ANOVA was followed by Tukey's test to compare the baseline and final values of each group whenever ANOVA detected a difference between the groups.

In addition, the frequency of adverse events was evaluated per visit for each treatment group according to the number and percentage of patients with any adverse event or by the type of adverse event being evaluated. A significance level of 5\% was adopted throughout the analysis.

\section{RESULTS}

As shown in the flowchart (Figure 1) from January 2008 until March 2009 was selected 964 women and of these, only 242 met the eligibility criteria to participate. They were divided in group A $(\mathrm{n}=80)$, group $\mathrm{B}(\mathrm{n}=80)$ and group $\mathrm{C}(\mathrm{n}=82)$. After the dropouts 66 women completed the study in group A, 54 in group B and 67 in group C.

No significant differences were found between the three treatment groups in regard to the baseline clinical and laboratory characteristics of the patients (Table 1).

In intention to treat analysis occurred a decreased significantly variation in TC, non-HDL and LDL cholesterol levels in group $\mathrm{B}(\mathrm{P}<0.05)$ and in the groups $\mathrm{A}$ and $\mathrm{C}(\mathrm{P}<0.0001)$. In Apo-B levels and Apo-B/Apo-A1 ratio a decreased significantly occurred only in groups $\mathrm{A}$ and C (Table 2).

In Table 2 Group A alone, mean Apo-A1 levels decreased $16.3 \mathrm{mg} / \mathrm{dl}$ at the final evaluation, a reduction of $10.4 \%$ compared to baseline $(\mathrm{P}<0.01)$ and the TG decrease $27.1 \mathrm{mg} / \mathrm{dl}(\mathrm{P}<0.05)$. A significant improvement in the cardiovascular markers Apo-B and the Apo-B/ Apo-A1 ratio at the end of the study in Group A (reductions of $33.2 \%$ and $25 \%$, respectively; $\mathrm{P}=0.0001)$ and in Group $\mathrm{C}$ (reductions of $26.7 \%, \mathrm{P}<0.0001$ and $33.3 \%, \mathrm{P}$ $<0.01$ respectively). There was no significant change in HDL in any of the groups.

Table 1. Baseline clinical and laboratory characteristics of the three treatment groups.

\begin{tabular}{ccccc}
\hline & & Groups & & \\
& A (n= 80) & B (n= 80) & C (n = 82) \\
\hline Variable & Mean \pm SD & Mean \pm SD & Mean \pm SD & P-value \\
Age (years) & $55 \pm 5$ & $55 \pm 5$ & $56 \pm 5$ & 0.58 \\
Weight (kg) & $67.2 \pm 10.6$ & $68.1 \pm 10.6$ & $66.7 \pm 10.4$ & 0.46 \\
Height (cm) & $154 \pm 7$ & $156 \pm 7$ & $154 \pm 6$ & 0.26 \\
BMI (kg/m) & $28.2 \pm 3.6$ & $28.0 \pm 3.5$ & $28.0 \pm 4.0$ & 0.72 \\
Waistcircumference (cm) & $98.2 \pm 9.5$ & $97.2 \pm 9.5$ & $96.6 \pm 9.8$ & 0.32 \\
TG (mg/dl) & $140.1 \pm 53.4$ & $152 \pm 77.5$ & $138.9 \pm 58.3$ & 0.32 \\
TC (mg/d) & $233.2 \pm 29$ & $234.0 \pm 31.2$ & $234.6 \pm 23.7$ & 0.95 \\
HDL-C (mg/d) & $55.6 \pm 17.2$ & $51.5 \pm 11.8$ & $55.0 \pm 13.6$ & 0.15 \\
Non-HDL cholesterol (mg/dL) & $177.6 \pm 33.6$ & $182.5 \pm 33.3$ & $179.5 \pm 28.1$ & 0.61 \\
LDL-C (mg/dl) & $152 \pm 27.0$ & $151.5 \pm 30.7$ & $151.8 \pm 27.7$ & 0.98 \\
Apo-A1 (mg/dl) & $156.6 \pm 32.8$ & $157.2 \pm 37.0$ & $152.6 \pm 34.8$ & $119.7 \pm 23.6$ \\
Apo-B (mg/dl) & $125.0 \pm 27.1$ & $125.5 \pm 32.2$ & $0.89 \pm 0.81$ & 0.65 \\
Apo-B/Apo-A1 & $0.83 \pm 0.23$ & $0.82 \pm 0.24$ & 0.33
\end{tabular}


Table 2. Intention to treat analyses of lipid and lipoprotein profile at baseline and final visit.

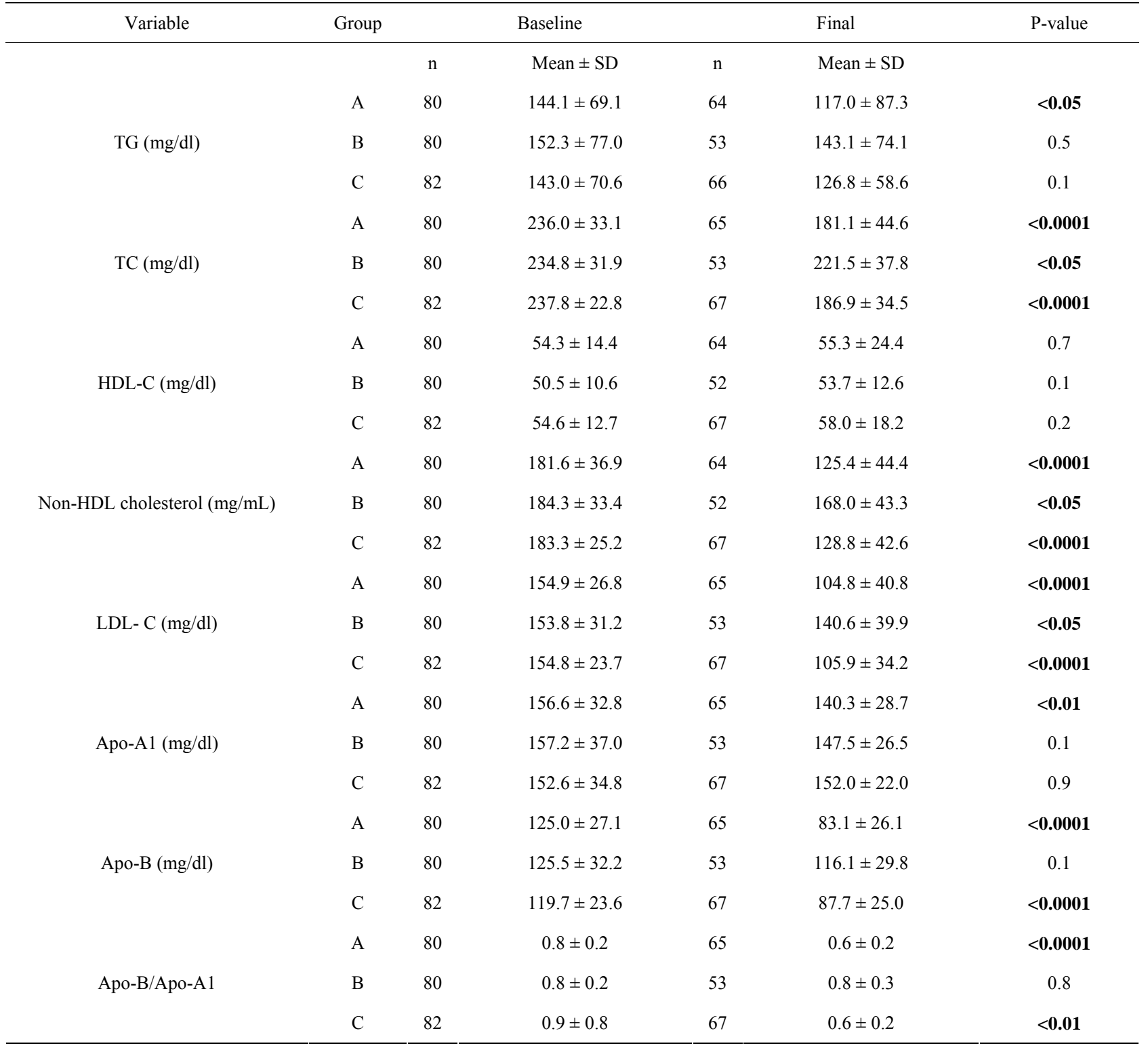

As shown in Figure 2, the treatment regimens used in Groups $\mathrm{A}$ and $\mathrm{C}$ were more effective than that used in Group B for reducing levels of TC, non-HDL cholesterol, LDL, Apo-B (A versus B, $\mathrm{P}<0.0001$ and $\mathrm{C}$ versus $\mathrm{B}, \mathrm{P}$ $<0.0001$ ) and the Apo-B/Apo-A1 ratio (A versus $\mathrm{B}, \mathrm{P}<$ 0.0001 and $C$ versus $B, P=0.005$ ). There was any significantly variation between the groups $\mathrm{A}$ and $\mathrm{C}$.

Mean TG, HDL and Apo A1 levels did not vary significantly between the groups. However for HDL and Apo $\mathrm{A} 1$ it almost reached statistical significance $(\mathrm{P}=$ 0.06) (Table 3).

In Group A, there was a mean reduction in HDL of $-2.27 \pm 12.35 \mathrm{mg} / \mathrm{dl}$, whereas in Group $\mathrm{C}$ a mean increase of $2.39 \pm 9.46 \mathrm{mg} / \mathrm{dl}$. The Apo-A1 cardiovascular risk marker decreased in both treatment groups; however, in Group A the mean reduction was $-13.91 \pm 28.93 \mathrm{mg} / \mathrm{dl}$ compared to $-1.77 \pm 33.77 \mathrm{mg} / \mathrm{dl}$ in Group C (Table 3).

A better response in the KI was found in Groups A and $\mathrm{B}$ compared to $\mathrm{C}$. The mean variations improvement in KI was already found at V2 when compared to baseline visit with a statistical significance between the groups (Figure 3).

Vaginal Bleeding was the most frequent adverse event affecting $28 \%$ of randomized patients. It happens more frequently in the groups using HT, 41 women in group A and 24 in group B. Breast pain was a complaint for $22.7 \%$ patients and also in the HT include groups. Nonspecific pain occurred in $11.5 \%$, mainly in the groups using Simvastatin. All other adverse events had a frequency less than $10 \%$. 


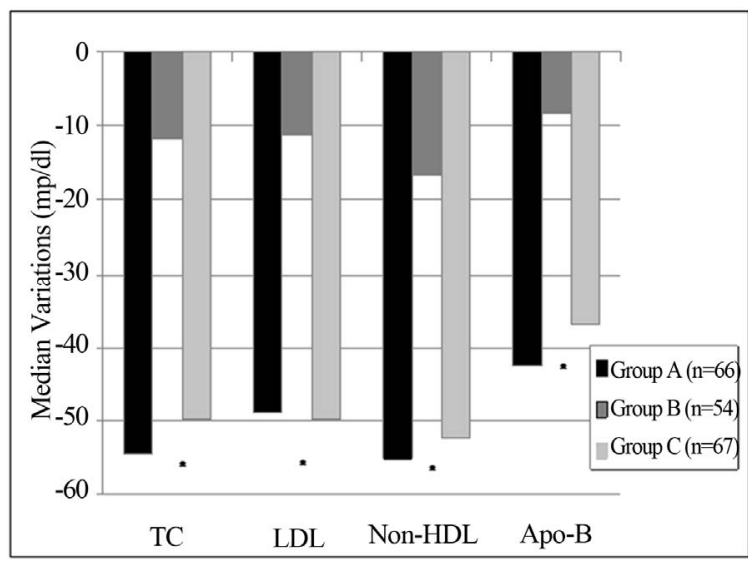

Figure 2. Median variations in TC, HDL-C, non-HDL cholesterol and Apo-B in per protocols analysis for each treatment group.

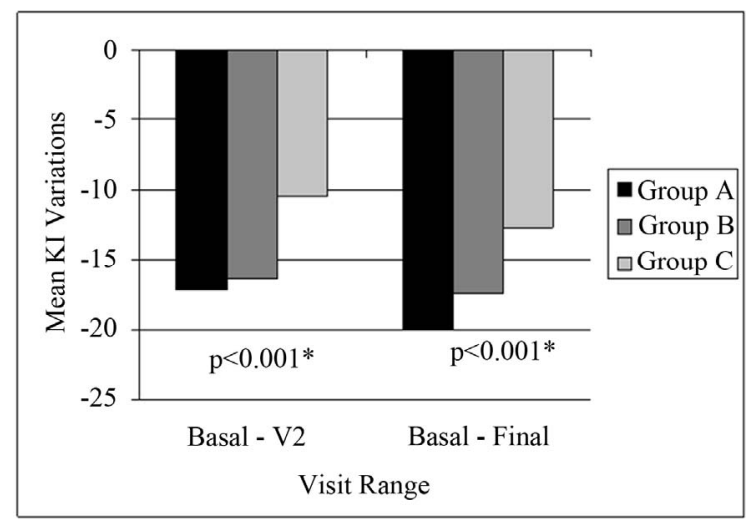

Figure 3. Evaluation of the mean KI variation throughout the study in the different groups.

\section{DISCUSSION}

Combined treatment with simvastatin and EPT has already been evaluated in other studies and reports on an improvement in lipid profiles have been convincing [15,19-21]. Prior to the Women's Health Initiative (WHI) study, EPT had come to be considered an alternative or even a first line therapy for the treatment of hypercholesterolemia in menopausal women $[13,14]$.

In view of the good therapeutic response achieved with this association and the concept of the lowest effective therapeutic dose that was established following the WHI study, [22] the protocol of this present trial was designed to evaluate the use of simvastatin with low-dose EPT for the first time.

In addition to having an abnormal lipid profile, all the groups in this sample population had BMI $>25 \mathrm{~kg} / \mathrm{m}^{2}$ and waist circumference $>88 \mathrm{~cm}$ at baseline, constituting a population at an increased risk of CVD. Nevertheless, since there was no statistically significant difference between the groups at baseline, these variables did not affect the final results.
Table 3. Comparison of the variations in lipid and lipoprotein profiles between the groups in the per protocol patients.

\begin{tabular}{|c|c|c|c|c|}
\hline Variable & Group & $\begin{array}{c}\text { Mean } \\
\text { Variation }\end{array}$ & SD & ANOVA \\
\hline & & $\mathbf{m g} / \mathbf{d L}$ & $\mathrm{mg} / \mathrm{dL}$ & $A$ vs $B$ vs $C$ \\
\hline \multirow{3}{*}{ TG (mg/dl) } & A & -20.90 & 73.86 & \multirow{3}{*}{0.74} \\
\hline & B & -18.31 & 61.97 & \\
\hline & $\mathrm{C}$ & -12.85 & 59.13 & \\
\hline \multirow{3}{*}{ TC (mg/dl) } & A & -54.35 & 43.92 & \multirow{3}{*}{$<0.0001^{*}$} \\
\hline & B & -11.75 & 27.84 & \\
\hline & $\mathrm{C}$ & -49.88 & 32.20 & \\
\hline \multirow{3}{*}{ HDL (mg/dl) } & A & -2.27 & 12.35 & \multirow{3}{*}{0.06} \\
\hline & B & 0.97 & 11.08 & \\
\hline & $\mathrm{C}$ & 2.39 & 9.46 & \\
\hline \multirow{3}{*}{$\begin{array}{c}\text { Non-HDL } \\
\text { cholesterol (mg/dl) }\end{array}$} & A & -55.18 & 43.97 & \multirow{3}{*}{$<0.0001^{*}$} \\
\hline & $\mathrm{B}$ & -16.59 & 35.86 & \\
\hline & $\mathrm{C}$ & -52.27 & 32.87 & \\
\hline \multirow{3}{*}{ LDL (mg/dl) } & A & -48.85 & 42.98 & \multirow{3}{*}{$<0.0001^{*}$} \\
\hline & $\mathrm{B}$ & -11.29 & 31.58 & \\
\hline & $\mathrm{C}$ & -49.71 & 33.16 & \\
\hline \multirow{3}{*}{ Apo-A1 (mg/dl) } & A & -13.91 & 28.93 & \multirow{3}{*}{0.06} \\
\hline & B & -8.33 & 27.47 & \\
\hline & $\mathrm{C}$ & -1.77 & 33.27 & \\
\hline \multirow{3}{*}{ Apo-B (mg/dl) } & A & -42.39 & 28.58 & \multirow{3}{*}{$<0.0001^{*}$} \\
\hline & $\mathrm{B}$ & -8.26 & 17.35 & \\
\hline & $\mathrm{C}$ & -36.92 & 25.64 & \\
\hline \multirow{3}{*}{ Аpo-B/Apo-A1 } & $\mathrm{A}$ & -0.23 & 0.21 & \multirow{3}{*}{$0.003^{*}$} \\
\hline & $\mathrm{B}$ & -0.01 & 0.14 & \\
\hline & C & -0.35 & 0.85 & \\
\hline
\end{tabular}

Group A n $=66$, Group B $n=54$ and Group C n $=67 ;{ }^{*}$ Tukey Kramer test not different for A X C.

In the group of women using hormone therapy alone (B), significant changes occurred in TC, non-HDL and LDL cholesterol variables evaluated. There was a reduction in TC and LDL levels of approximately $5 \%$ and $8 \%$, respectively. This positive effect on lipid profiles is in agreement with reports in the literature. Samsioe et al. evaluated the lipid profile of women using the same dose and type of hormone therapy and reported a reduction of $10.6 \%$ and $11.3 \%$ in TC and LDL, respectively [20]. The WHI study, which did not endorse EPT for the prevention of CVD, showed an improvement of $12.7 \%$ in LDL levels [6]. 
The variation in HDL and Apo-A1 in the group of women using EPT alone was not statistically significant in the present study. This is also in agreement with data published in the literature from studies in which NETA appeared to cancel out the effect of oral estrogen in increasing HDL levels [20,23]. On the other hand, when simvastatin was associated with EPT, significant improvements were found in serum levels of TC, LDL, nonHDL cholesterol, Apo-B and in the Apo-B/Apo-A1 ratio after 16 weeks of treatment. As expected, this also occurred in the group of women using simvastatin alone (C).

Comparison between groups shows that when EPT was associated with simvastatin, the benefits found with the use of simvastatin alone were preserved, thereby confirming the potential of this therapeutic combination for the possible prevention of CVD in women with menopausal symptoms.

The Scandinavian Simvastatin Survival Study (4S) was the first to evaluate the use of $20 \mathrm{mg}$ of simvastatin for the secondary prevention of CVD. The results of this study showed a reduction of $42 \%$ in deaths from cardiovascular events compared to the placebo group [12]. Considering that in the present study, the association of EPT with simvastatin resulted in similar effects to those found with the use of simvastatin alone, it is reasonable to conclude that this combination would also have a cardioprotective effect.

Increased LDL levels represent a risk factor for CVD. Based on observational studies, a $1 \%$ reduction in LDL levels implies a $2 \%$ reduction in the risk of CVD [23]. In the present study, the users of EPT and simvastatin had a reduction of over $30 \%$ in this lipid.

Levels of Apo-A1, an important component of HDL, decreased only in the treatment groups using EPT at the end of the study compared to baseline. In the group of women using simvastatin alone, this decrease was found to be irrelevant. This different behavior might explain the significant statistically trend of this variable in the between group comparison.

Comparisons between groups in HDL also show a trend of significance. In intention to treat's analysis all groups had an increase in HDL levels. This was not repeated in the evaluation of patients per protocols in which the group A showed a reduction while the other groups an increase. However, mean HDL levels failed to fall below $50 \mathrm{mg} / \mathrm{dl}$, the value considered representative of a risk of CVD, at any point in the study and in any of the groups evaluated $[25,26]$.

The explanation for the different behavior in HDL and Apo-A1 in the HT plus simvastatin treatment group is complex. It may be related to use of NETA. As previously mentioned, studies with a high level of evidence have shown that the combination of NETA with estrogen may reduce or cancel the effect of this hormone, particu- larly on the HDL fraction of cholesterol [19,22,27]. This is a consequence of the drug's androgenic profile and appears to be directly related to the dose used. The present study failed to contribute towards clarifying whether the effect derives from the progestogen or not, since there was no group treated with EPT that did not contain this progestogen or that included different progestogens.

Recent studies have shown that the Apo-B/Apo-A1 ratio is a good predictor of risk of myocardial infarction and CVD, constituting a better indicator than the measurement of triglycerides and HDL alone [28-30]. In addition to this index, Apo-B levels alone have also been shown to be significantly associated with an increased risk of CVD [26,29]. A statistically significant reduction was found in these two indicators, both in the users of simvastatin alone and in the group of women using EPT + simvastatin.

There was no statistically significant difference between the association of EPT and simvastatin and EPT without concomitant use of simvastatin with regard to the improvement of menopausal symptoms. Therefore, it is reasonable to presume that simvastatin did not reduce the effects of HRT on these symptoms.

The reduction in the KI in the group using simvastatin alone (C) may be a result of the placebo effect. A metaanalysis conducted by the Cochrane Library in 2004 involving 24 clinical trials found that women using placebo had a reduction of over $50 \%$ in the number of hot flashes [31]. No study in the literature has shown the actual benefit of statins in treating any of the symptoms of the menopause.

The main adverse events were related to the use of hormone therapy as vaginal bleeding and breast pain. These symptoms are common in women using hormone therapy and may be one reason for the greater number of patients discontinued in group B. Nonspecific pain occurred more in the group using simvastatin alone. Apart from these three adverse events the others were often less than $10 \%$.

Finally, the present study was limited to evaluating EPT that consisted of one single compound given by one single route of administration. There is a need to evaluate the effect of the association of simvastatin with other EPT regimens and using other routes of administration, including the use of other progestogens with metabolic profiles that differ from that of norethisterone.

Cardiovascular risk is related to clinical conditions such as BMI, waist circumference, smoking, a sedentary lifestyle and type of diet. The present study failed to control for any of these variables; however, the groups were similar at baseline with respect to all possible confounding variables. Another limitation is the fact that intermediary risk markers rather than the occurrence of cardiovascular events were evaluated. 
One of the goals of hormone treatment in women with menopausal symptoms is to improve their quality of life and this was not one of the objectives of this study; therefore, the present results fail to provide any answer regarding whether the association of EPT and simvastatin has the same effect on quality of life as the use of EPT alone.

\section{CONCLUSION}

In conclusion, the association of E2/NETA low-dose simvastatin relieved climacteric symptoms similar to that observed with isolated E2/NETA and improved lipid and lipoprotein profile similar to the isolated use of simvastatin in, postmenopausal women with dyslipidemia. The use of E2/NETA without simvastatin decreased menopausal symptoms, but it was not as effective as the other treatments in improving dyslipidemia.

\section{ACKNOWLEDGEMENTS}

Priscilla Scaff Padilha, Biolab clinical research coordinator.

\section{REFERENCES}

[1] Mosca, L., Collins, P., Herrington, D.M., et al. (2001) Hormone replacement therapy and cardiovascular disease: A statement for health care professionals from the American Heart Association. Circulation, 104, 499-503. doi:10.1161/hc2901.092200

[2] Herrington, D.M., Reboussin, D.M., Brosnihan, K.B., et al. (2000) Effects of estrogen replacement on the progression of coronary-artery atherosclerosis. New England Journal of Medicine, 343, 522-529. doi:10.1056/NEJM200008243430801

[3] Humphrey, L.L., Chan, B.K. and Sox, H.C. (2002) Postmenopausal hormone replacement therapy and the primary prevention of cardiovascular disease. Annals of Internal Medicine, 137, 273-284. doi:10.7326/0003-4819-137-4-200208200-00012

[4] Grady, D., Rubin, S.M., Petitti, D.B., et al. (1992) Hormone therapy to prevent disease and prolong life in postmenopausal women. Annals of Internal Medicine, 117, 1016-1037. doi:10.7326/0003-4819-117-12-1016

[5] Hulley, S., Grady, D., Bush, T., et al. (1998) Randomized trial of estrogen plus progestin for secondary prevention of coronary heart disease in postmenopausal women. Heart and Estrogen/progestin Replacement Study (HERS) Research Gorup. JAMA, 280, 605-613. doi:10.1001/jama.280.7.605

[6] Manson, J.E., Hsia, J., Johnson, K.C., et al. (2003) Estrogen plus progestin and the risk of coronary heart disease. New England Journal of Medicine, 349, 523-534. doi:10.1056/NEJMoa030808

[7] Rossouw, J.E. (2006) Implications of recent clinical trials of postmenopausal hormone therapy for management of cardiovascular disease. Annals of the New York Academy of Sciences, 1089, 444-453.

\section{doi:10.1196/annals.1386.046}

[8] LaRosa, J.C., He, J. and Vupputuri, S. (1999) Effect of statins on risk of coronary disease: A meta-analysis of randomized controlled trials. JAMA, 282, 2340-2346. doi:10.1001/jama.282.24.2340

[9] Downs, J.R., Clearfield, M., Weis, S., et al. (1998) Primary prevention of acute coronary events with lovastatin in men and women with average cholesterol levels: Results of AFCAPS/TexCAPS. Air Force/Texas Coronary Atherosclerosis Prevention Study. JAMA, 279, 1615-1622. doi:10.1001/jama.279.20.1615

[10] Herd, J.A., Ballantyne, C.M., Farmer, J.A., et al. (1997) Effects of fluvastatin on coronary atherosclerosis in patients with mild to moderate cholesterol elevations (Lipoprotein and Coronary Atherosclerosis Study [LCAS]). American Journal of Cardiology, 80, 278-286. doi:10.1016/S0002-9149(97)00346-9

[11] Heart Protection Study Collaborative Group (2002) MRC/ BHF heart protection study of cholesterol lowering with simvastatin in 20,536 high-risk individuals: A randomized placebo-controlled trial. Lancet, 360, 7-22. doi:10.1016/S0140-6736(02)09327-3

[12] (1994) Randomised trial of cholesterol lowering in 4444 patients with coronary heart disease: The Scandinavian Simvastatin Survival Study (4S). Lancet, 344, 1383-1385.

[13] Darling, G.M., Johns, J.A., McCloud, P.I. and Davis, S.R. (1999) Concurrent use of simvastatin and estrogen-progestin therapy compared with each therapy alone for hypercholesterolemia in postmenopausal women. Climacteric, 2, 181-188. doi:10.3109/13697139909038060

[14] Vigna, G.B., Donegà, P., Zanca, R., et al. (2002) Simvastatin, transdermal patch, and oral estrogen-progestogen preparation in early-postmenopausal hypercholesterolemic women: A randomized, placebo-controlled clinical trial Metabolism, 51, 1463-1470. doi:10.1053/meta.2002.35584

[15] Fak, A.S., Erenus, M., Tezcan, H., et al. (2000) Effects of simvastatin only or in combination with continuous combined hormone replacement therapy on serum lipid levels in hypercholesterolaemic post-menopausal women. European Heart Journal, 21, 190-197. doi:10.1053/euhj.1999.1582

[16] Darling, G.M., Johns, J.A., McCloud, P.I. and Davis, S.R. (1997) Estrogen and progestin compared with simvastatin for hypercholesterolemia in postmenopausal women. New England Journal of Medicine, 337, 595-601. doi:10.1056/NEJM199708283370903

[17] Hsia, J., Langer, R.D., Manson, J.E., et al. (2006) Conjugated equine estrogens and coronary heart disease: The Women's Health Initiative. Archives of Internal Medicine, 166, 357-365. doi:10.1001/archinte.166.3.357

[18] Dupont, W.D. and Plummer, W.D. (1997) PS power and sample size program available for free on the internet. Control Clinical Trials, 18, 274. doi:10.1016/S0197-2456(97)00074-3

[19] Davis, S.R., Goldstat, R., Newman, A., et al. (2002) Differing effects of low-dose estrogen-progestin therapy and pravastatin in postmenopausal hypercholesterolemic women. Climacteric, 5, 341-350. 
[20] Samsioe, G., Li, C., Borgfeldt, C., et al. (2002) Changes in lipid and lipoprotein profile in postmenopausal women receiving low-dose combinations of 17 beta-estradiol and norethisterone acetate. Menopause, 9, 335-342. doi:10.1097/00042192-200209000-00006

[21] Koh, K.K., Cardillo, C., Bui, M.N., et al. (1999) Vascular effects of estrogen and cholesterol-lowering therapies in hypercholesterolemic postmenopausal women. Circulation, 99, 354-360. doi:10.1161/01.CIR.99.3.354

[22] Rossouw, J.E., Prentice, R.L., Manson, J.E., Wu, L., Barad, D., Barnabei, V.M. et al. (2007) Postmenopausal hormone therapy and risk of cardiovascular disease by age and years since menopause. JAMA, 297, 1465-1477. doi:10.1001/jama.297.13.1465

[23] Davidson, M.H., Maki, K.C., Marx, P., et al. (2000) Effects of continuous estrogen and estrogen-progestin replacement regimens on cardiovascular risk markers in postmenopausal women. Archives of Internal Medicine, 160, 3315-3325. doi:10.1001/archinte.160.21.3315

[24] The Lipid Research Clinics Coronary Primary Prevention (1984) Trial results: I. Reduction in incidence of coronary heart disease. JAMA, 251, 351-364. doi:10.1001/jama.1984.03340270029025

[25] Expert Panel on Detection, Evaluation, and Treatment of High Blood Cholesterol in Adults (2001) Executive Summary of the Third Report of the National Cholesterol Education Program (NCEP) Expert Panel on Detection, Evaluation, and Treatment of High Blood Cholesterol in Adults (Adult Treatment Panel III). JAMA, 285, 24862497. doi:10.1001/jama.285.19.2486
[26] Ingelsson, E., Schaefer, E.J., Contois, J.H., et al. (2007) Clinical utility of different lipid measures for prediction of coronary heart disease in men and women. JAMA, 298, 776-785. doi:10.1001/jama.298.7.776

[27] Al-Azzawi, F., Wahab, M., Sami, S., et al. (2004) Randomized trial of effects of estradiol in combination with either norethisterone acetate or trimegestone on lipids and lipoproteins in postmenopausal women. Climacteric, 7 , 292-300. doi:10.1080/13697130400001364

[28] Walldius, G. and Jungner, I. (2006) The apoB/apoA-I ratio: A strong, new risk factor for cardiovascular disease and a target for lipid-lowering therapy-A review of the evidence. Journal of Internal Medicine, 259, 493-519. doi:10.1111/j.1365-2796.2006.01643.x

[29] Yusuf, S., Hawken, S., Ounpuu, S., et al. (2004) Effect of potentially modifiable risk factors associated with myocardial infarction in 52 countries (the Interheart study): Case-control study. Lancet, 364, 937-952. doi:10.1016/S0140-6736(04)17018-9

[30] Sierra-Johnson, J., Fisher, R.M., Romero-Corral, A., et al. (2009) Concentration of apolipoprotein B is comparable with the apolipoprotein B/apolipoprotein A-I ratio and better than routine clinical lipid measurements in predicting coronary heart disease mortality: Findings from a multi-ethnic US population. European Heart Journal, 30, 710-717. doi:10.1093/eurheartj/ehn347

[31] Maclennan, A.H., Broadbent, J.L., Lester, S. and Moore, V. (2004) Oral oestrogen and combined oestrogen/progestogen therapy versus placebo for hot flushes. Cochrane Database of Systematic Reviews, 4, CD002978.

\section{ABREVIATIONS LIST}

EPT-EstrogenplusProgestentherapy

E2-Estradiol

NETA - norethisterone

CDV_-Cardiovascular disease

HT-Hormonetherapy

HRT - HormoneReplacementTherapy

BMI-Body Mass Index
$\mathrm{KI}$-Kupperman Index

TC_-Total Cholesterol

LDL_Low density lipoprotein

TG-Triglycerides

HDL-High density lipoprotein

ApoB-Apolipoprotein B

Apo A1-Apoliprotein A1 